\title{
Efficient Networks in Models with Player and Partner Heterogeneity*
}

\author{
Emre Unlu ${ }^{\dagger}$
}

March 8, 2017

\begin{abstract}
This paper characterizes efficient networks in player and partner heterogeneity models for both the two-way flow and the one-way flow networks. Player (partner) dependent network formation allows benefits and costs to be player (partner) heterogeneous, which is an important extension for modeling social networks in the real world. This paper shows that efficient networks are minimally connected and have either a star-type or a variation of star type architectures in the two-way flow model, and have the wheel architecture in the one-way flow model.
\end{abstract}

Keywords: Efficiency, Networks, Player and Partner Heterogeneity

JEL Classification: C72, D85

*I would like to thank an editor and two anonymous referees, Pascal Billand, Christophe Bravard, Francis Bloch, Surajit Bortokokey, Sudipta Sarangi, Matt Wiser and the participants of PET11 and Games 2012 conferences for very helpful discussions and comments.

${ }^{\dagger}$ Business School, Edge Hill University, St Helens Road, Ormskirk, Lancashire; L39 4QP United Kingdom; e-mail: unlue@edgehill.ac.uk. 


\section{Introduction}

A growing literature on social and economic networks addresses dominant effect of networks on various important outcomes such as labor markets, the spread of diseases, education and crime. Hence vast research-not only in economics, but also in other disciplines has been conducted to understand how the networks emerge and how they evolve over time. A central theme in the literature on network formation is the conflict between the set of stable networks and the set of efficient networks. A substantial literature focuses on individual optimization through strict Nash networks, where no agent can make herself better off by deviating from her current strategy, given the strategies of the other players. Even though (strict) Nash networks are very well studied, there have been few studies about efficiency. This paper fills the gap in the literature of network formation by describing the efficient network architectures with heterogeneous agents, and addresses the issue of efficiency in the form of maximizing the sum of the utilities of the players in the network and compare the architectures of efficient networks with (strict) Nash networks.

The seminal papers on network formation are Jackson and Wolinsky (1996) and Bala and Goyal (2000). Jackson and Wolinsky (1996) provide two-sided link formation, where the cost of forming links is shared by the participants and introduce pairwise stability. They show that there is a substantial conflict between pairwise stable and efficient networks. Bala and Goyal (2000) provide a theoretical framework to address network formation in a non-cooperative setting with homogeneous players, where the cost of forming links is on one side. They discuss two different types of flow. In the two-way flow model, the network is undirected, so the two players participating in a link can access benefits from each other. In the one-way flow model, the network is directed; hence only the initiating player can access the benefits of the link. This paper follows the Bala and Goyal (2000) framework, where link costs are levied only by the person initiating the link.

Galeotti et al. (2006) relax the homogeneity assumption in the two-way flow model, so the benefits from a link and the cost of sponsoring a link are player dependent, meaning that it only depends on who forms the link. Galeotti et al. (2006) conclude that Nash and efficient networks coincide under linear payoff situations; however, this study shows that with more general payoff function specifications this result does not always hold. Galeotti (2006) extends the one-way flow model by allowing benefits and costs to be player and partner heterogeneous. In a one-way flow model, only the node who sponsors a 
link accesses the benefit. This fact yields a wheel type efficient architecture, that coincides with strict Nash network architecture.

Billand et al. (2011) introduce partner heterogeneity in the two-way flow model, where the benefit and cost of making a link are partner heterogeneous, meaning that it depends only upon whom is being accessed in terms of the benefits and costs. Although Nash networks are clearly identified under heterogeneity, not much has been done about efficient networks under heterogeneity.

The model starts with a general payoff function in a player heterogeneity model, satisfying common assumptions in the literature. For instance, the player's payoffs are assumed to be strictly increasing with respect to the benefits and strictly decreasing with respect to the costs of forming links. However, under these common assumptions, efficient networks can still have maximal diameter and it is not possible to characterize the architectures. Similar arguments can be made for the partner heterogeneous model. When there is heterogeneity between players, the efficient network architectures depend on four factors: (i) the value of players (benefit obtained by linking to each player); (ii) the number of minimum cost players; (iii) the difference in cost between the minimum cost player(s) and the other players; and (iv) the functional form of payoffs. The first factor is controlled by a restriction which ensures that all links are profitable. To deal with the third factor, the widely used linearity, strict concavity and convexity assumptions are introduced on the payoffs. The fourth factor is accounted for with the restrictions that are already imposed, but an additional condition is imposed in the two-way flow for each model. Since the heterogeneity in the values only affects the connectivity of the networks and only the connected networks are considered in this paper, the values that are obtained from forming links are assumed to be homogeneous for all players and the heterogeneity is allowed for only cost of link formation. This assumption simplifies the complexity of the efficient networks and enables to focus on costs of link formation.

The architecture as well as the diameter of the efficient networks are given in the paper. The architecture provides information about how the efficient networks look, and the diameter helps to determine the maximum distance between any two players in the network. The crucial difference between player and partner heterogeneity models is in the change of the player who sponsors the links. However, there are also slight differences in architecture and diameter.

The rest of the paper is organized as follows: Section 2 presents the basics of the model setup and 
some important definitions. Section 3 identifies the efficient networks in a player heterogeneity model. Section 4 examines the efficient networks in the partner heterogeneity model. The paper concludes with a discussion and comparison of differences in efficient network structures.

\section{Model Setup}

$N=\{1, \ldots, n\}$ denotes the set of players. A directed network $\mathbf{g}=(N, A)$ is a pair of sets: the set $N$ of nodes and the set $A \subset N \times N$ of arcs. $A=A(\mathrm{~g})$ corresponds to the links between players. The undirected counterpart of $\mathbf{g}, \overline{\mathbf{g}}$ is obtained by ignoring the orientation of arcs of $\mathbf{g}$ and treating links between the same players as a single link.

Each player $i$ chooses a strategy $\mathbf{g}_{i}=\left(g_{i, 1}, \ldots, g_{i, i-1}, g_{i, i+1}, \ldots, g_{i, n}\right)$ where $g_{i, j} \in\{0,1\}$ for all $j \in N \backslash\{i\}$. The interpretation of $g_{i, j}=1$ is that player $i$ forms a link with player $j \neq i$, and the interpretation of $g_{i, j}=0$ is that $i$ forms no link with player $j$. A link between player $i$ and $j$ can allow for either one-way(asymmetric) or two-way(symmetric) flow of information. With one-way communication, the link $g_{i, j}=1$ enables player $i$ to access $j$ 's information but not vice-versa. With two-way communication, $g_{i, j}=1$ allows both $i$ and $j$ to access each other's information. It is assumed that player $i$ cannot form a link with herself. Only pure strategies are considered. Let $\mathcal{G}_{i}$ be the set of all strategies of player $i \in N$. If $g_{i, j}=1$, then by definition $j i \in A(\mathbf{g})$. A link $j i$ is shown by an arrow from $j$ to $i$. Thus, if the player $i$ chooses to link with $j$, the arc will be directed from $j$ to $i$ and it also means that player $i$ incurs the cost of the link formation.

For a directed network, g, a path from player $k$ to player $j, j \neq k$, is a finite sequence $j_{0}, j_{1}, \ldots, j_{m}$ of distinct players such that $j_{0}=j, j_{m}=k$ and $\mathbf{g}_{j_{\ell}, j_{\ell+1}}=1$ for $\ell=0, \ldots, m-1$. A chain exists between player $k$ and player $j, j \neq k$ by replacing $g_{j_{\ell}, j_{\ell+1}}=1$ by $\max \left\{g_{j_{\ell}, j_{\ell+1}}, g_{j_{\ell+1}, j_{\ell}}\right\}=1$.

Given a network $\mathbf{g}$, a component $C(\mathbf{g})$ of $\mathbf{g}$ is defined as a set of players such that there is a chain between any two players who belong to $C(\mathbf{g})$ and there does not exist a chain between a player in $C(\mathbf{g})$ and a player who does not belong to $C(\mathbf{g})$. A network $\mathbf{g}$ is said to be connected if it contains one component and all players belong to this component. Finally, a network is minimally connected if it is not possible to preserve its connectivity whenever a link is removed.

Definition 1: Let $l_{i j}$ denote the (geodesic) distance from players $i$ to $j$. Then the diameter $D(g)$ of a 
network $g$ is the maximum distance $l_{i j}$ over player pairs $i j$.

A network $\mathbf{g}$ is a star if there is a player $i$ such that $\max \left\{g_{i, j}, g_{j, i}\right\}=1$ for all $j \in N \backslash\{i\}$ and $g_{\ell, j}=0$ for all $\ell \in N \backslash\{i\}$ and $j \in N \backslash\{i, \ell\}$. The network $\mathbf{g}$ is an inward pointing star or center-sponsored star if it is a star and for the center player $i, g_{j, i}=0$ for all $j \in N \backslash\{i\}$. The network $\mathbf{g}$ is an outward pointing star or periphery-sponsored star if it is a star and for the center player $i, g_{i, j}=0$ for all $j \in N \backslash\{i\}$.

A network in which each group constitutes a star and a central player $i$ of group $l$ forms a link with the central player $j$ of group $l^{*}$ where $l \neq l^{\prime}$, is referred to as an interlinked star network. If each star is center-sponsored (periphery-sponsored), the network is said to be an interlinked center-sponsored (periphery-sponsored) star. A player is called a bridge player if she has a link to a center of at least two star architectures. A generalized interlinked star network is an interlinked star network where there is a bridge player between the center of each star.

Let $N_{i}(\mathbf{g})$ be the set of players observed by player $i$ in the network $\mathbf{g}$. In the two-way flow model, $j \in N_{i}(\mathbf{g})$ whenever there is a chain between $i$ and $j$ in $\mathbf{g}$. In the one-way flow model, $j \in N_{i}(\mathbf{g})$ whenever there is a path from $j$ to $i$ in $\mathbf{g}$. Sometimes, to simplify notation, the number of links formed by player $i$ in network $\mathbf{g}^{0}$ will be denoted by $N_{i}^{0}$ instead of $N_{i}\left(\mathbf{g}^{0}\right)$. Moreover, the network identical to $\mathbf{g}$, except that the link $i j$ is added (deleted) will be denoted as $\mathbf{g}+i j(\mathbf{g}-i j)$.

The following two classes of model assume that the costs of a link can be different among players. In the player heterogeneity model proposed by Galeotti et al. (2006), each player $i$ obtains $V>0$ from each player $j \in N_{i}(\mathbf{g})$, and incurs a cost $c_{i}>0$ when she forms a link with player $j \in N_{i}(\mathbf{g})$. In the partner heterogeneity model introduced by Billand et al. (2011), each player $i$ obtains $V>0$ from each player $j \in N_{i}(\mathbf{g})$, and incurs a cost $c_{j}>0$ when she forms a link with a player $j \in N_{i}(\mathbf{g})$. In other words, in the player heterogeneity model, the cost of sponsoring a link for player $i$ to player $j$ depends on player $i$ 's characteristics, while in the partner heterogeneity model, the cost of sponsoring a link for a player $i$ to a player $j$ depends on $j$ 's characteristics.

Let $\pi: \mathbf{g} \mapsto \pi(\mathbf{g})$ be the payoffs of player $i$ in the network $\mathbf{g}$ and $\phi: \mathbb{R}^{2} \rightarrow \mathbb{R}$ be a function. In the player heterogeneity model, the payoff function of a player $i$ is given by

$$
\pi_{i}(\mathbf{g})=\phi\left(\sum_{j \in N_{i}(\mathbf{g})} V, \sum_{j \in N} \mathbf{g}_{i, j} c_{i}\right)
$$


while in the the partner heterogeneity model, the payoff function of a player $i$ is given by:

$$
\pi_{i}(\mathbf{g})=\phi\left(\sum_{j \in N_{i}(\mathbf{g})} V, \sum_{j \in N} \mathbf{g}_{i, j} c_{j}\right)
$$

Given the properties of the function $\phi$, the first term $x$ can be interpreted as the "benefits" that player $i$ receives from her links, while the second term $y$ measures the "costs" associated with forming them. It is assumed that $\phi(x, y)$ is increasing in $x$ and decreasing in $y$.

The strictly convex and strictly concave payoff functions in the cost argument are defined as the following, respectively.

Definition 2: Suppose $c_{1}, c_{2} \in\left(0, \max _{i \in N} c_{i}\right), c_{1}<c_{2}$, and $\kappa \in\left(0, c_{1}\right]$. If $\phi\left(V, c_{1}\right)-\phi\left(V, c_{1}-\kappa\right)<$ $\phi\left(V, c_{2}\right)-\phi\left(V, c_{2}-\kappa\right)$ then the payoff function is strictly convex in the cost argument.

Definition 3: Suppose $c_{1}, c_{2} \in\left(0, \max _{i \in N} c_{i}\right), c_{1}<c_{2}$, and $\kappa \in\left(0, c_{1}\right]$. If $\phi\left(V, c_{1}\right)-\phi\left(V, c_{1}-\kappa\right)>$ $\phi\left(V, c_{2}\right)-\phi\left(V, c_{2}-\kappa\right)$ then the payoff function is strictly concave in the cost argument.

The following definitions are used throughout the paper.

Definition 4: Let $S_{\min }$ be the set of players who have the minimum cost of forming a link in network $g$. Then, $S_{\text {min }}=\left\{j \in N: j \in \arg \min _{l \in N} c_{l}\right\}$. Define $n_{\text {min }}$ as the number of minimum cost players in network $g$ so that $n_{\text {min }}=\left|S_{\text {min }}\right|$.

Definition 5: Let $S_{\max }$ be the set of players who have the highest cost of forming a link in network $g$. Then, $S_{\text {max }}=\left\{j \in N: j \in \arg \max _{l \in N} c_{l}\right\}$. Define $n_{\max }$ as the number of the highest cost players in network $g$ so that $n_{\max }=\left|S_{\max }\right|$.

For simplicity it is assumed that the benefits that are obtained from forming links are the same for each player and the heterogeneity exists for cost of link formation within the players. Hence, it follows that, if network $\mathbf{g}$ is connected, then each player obtains a benefit equal to $\bar{V}=n V$.

Given a network $\mathbf{g}$, the aggregate payoff is defined by: $W(\mathbf{g})=\sum_{i=1}^{n} \pi_{i}(\mathbf{g})$. A network is said to be efficient if $W(\mathbf{g}) \geq W\left(\mathbf{g}^{\prime}\right)$ for any network $\mathbf{g}^{\prime} \neq \mathbf{g}$. 


\section{$3 \quad$ Player Heterogeneity Models}

\subsection{Player Heterogeneity in the Two-way Flow Model}

\subsubsection{General Payoff Function}

In this section, the efficient network architectures with heterogeneous players in two-way flow information is studied. The payoff function is given by (1). Note that the payoff function does not assume any particular functional form and it does not impose any restriction on the cost of forming links. One immediate result is that the set of efficient networks is very large. In particular, there might be some players who are not connected to the other players; in that case, the diameter of the efficient network goes to infinity. However, the following proposition shows that it is possible to obtain minimal networks as the efficient networks.

Proposition 1: Suppose the payoff function is given by (1). Then, any efficient network $\mathbf{g}$ is minimal.

Proof: Suppose the network $\mathbf{g}$ is not minimal. Then, there is a minimal network $\mathbf{g}^{\prime}$ that allows each player to obtain the same total benefits and involves fewer links. Hence $\mathbf{g}$ is not an efficient network. This results in a contradiction.

The above proposition shows that the players access each other with a minimum number of links in the efficient networks. This indicates that if players $i$ and $j$ are connected in the efficient network, there will be only one path from player $i$ to $j$. This means that any redundant links are eliminated to maximize the aggregate payoff of the players. It follows that the efficient networks are minimal in a two-way flow player heterogeneity model. Note that this result also holds for a two-way flow partner heterogeneity model. To ensure connectivity in the efficient network, the following assumption is imposed.

A1: $\phi\left(x+V, y+g_{i j} c_{i}\right) \geq \phi(x, y)$, for all $i \in N$.

A1 eliminates the efficient networks that contain singletons or disconnected components. Once A1 is satisfied, efficient networks are connected.

Lemma 1: Suppose the payoff function is given by (1) and A1 is satisfied. Then, any efficient network, $\mathrm{g}$ is minimally connected. 
Proof: Suppose not. Then there exists an efficient network, $\mathbf{g}^{\prime}$ with two components $C_{1}\left(\mathbf{g}^{\prime}\right)$ and $C_{2}\left(\mathbf{g}^{\prime}\right)$. Consider two players $k$ and $m$ such that $k \in C_{1}\left(\mathbf{g}^{\prime}\right)$ and $m \in C_{2}\left(\mathbf{g}^{\prime}\right)$. Let $\mathbf{g}$ be a network such that $\mathbf{g}=\mathbf{g}^{\prime}+k m$. Then, by A1, it follows that $W(\mathbf{g})>W\left(\mathbf{g}^{\prime}\right)$. Therefore $\mathbf{g}^{\prime}$ cannot be an efficient network and the proof follows. From the above contradiction, if A1 is satisfied, then the efficient networks do not have any singletons or disconnected components. As shown in Proposition 1, to maximize the aggregate payoff any redundant links in the network are eliminated. Combining these results, it can be argued that the efficient networks are minimally connected.

The next proposition indicates that when the benefits and costs of link formation are allowed to vary freely, it is possible to have decentralized architectures and even maximal diameter.

Proposition 2: Suppose the payoff function is given by (1) and satisfies A1. There exists parameter values $V$ and $c_{i}$ such that the efficient network, $\mathbf{g}$, has maximum diameter, $(n-1)$.

Formal proof is omitted. To illustrate an efficient network, which has maximal diameter, the following example which utilizes an additively separable form, where the benefits and costs of link formation can be expressed as separate terms ${ }^{1}$ is provided.

Example 1: Suppose the payoff function of player $i$ is given by:

$$
\pi_{i}(\mathbf{g})=\left(\sum_{j \in N_{i}(\mathbf{g})} V\right)^{1 / 2}-\left(\sum_{j \in N} g_{i, j} c_{i}\right)^{2}
$$

Suppose $n=4$. Moreover, suppose there is one minimum cost player represented by $\mathrm{L}$ and 3 high cost players represented by $\mathrm{H}$. Let $c_{L}$ and $c_{H}$ be equal to 2 and 3 respectively ${ }^{2}$. Assume that $V=100$. Note that A1 holds for these parameter values. Now suppose that all links are sponsored by L, which can be represented by an inward pointing star. For this case, $\pi_{L}=\sqrt{400}-(3 \times 2)^{2}=-16$ and $\pi_{H}=\sqrt{400}$ which implies $W\left(\mathbf{g}^{\prime}\right)=\sum_{i} \pi_{i}\left(\mathbf{g}^{\prime}\right)=44$. Compare this with the efficient network $\mathbf{g}$, where each player sponsors a single link except one $\mathrm{H}$ type player. For this case, $\pi_{L}=16$ and $\pi_{H}=20$ for the $\mathrm{H}$ type player who does not sponsor any link and $\pi_{H}=11$ for the ones who sponsors a single link. This yields

\footnotetext{
${ }^{1}$ Here, an additively separable form which has economic interpretation and easy to construct is considered. The below function satisfies the assumptions of being strictly increasing in value and strictly decreasing in cost.

${ }^{2}$ For simplicity, two types of players represented by $\mathrm{L}$ and $\mathrm{H}$ are introduced. However, it is possible to have any heterogeneity between players in the costs of sponsoring links.
} 
$W(\mathbf{g})=58$. This example shows that the diameter can be as high as $(n-1)$ depending on the payoff function. Due to general payoff function specification, it cannot be ensured that all the links will be sponsored by the minimum cost player in the efficient network. After sponsoring a single link, the marginal cost of sponsoring another link to the minimum cost player is $16-4=12$. However, the marginal cost of sponsoring a single link for a high cost player is $3^{2}=9$.

The above example provides the intuition to determine the factors that affect the architecture of the efficient networks. It also demonstrates that strict concavity or convexity of the payoff function in terms of cost plays a role in determining the diameter of the efficient network. ${ }^{3}$ Therefore, restrictions on the payoff function are imposed. This study considers the following cases: linear payoffs, and strictly convex and concave payoffs in the cost argument.

\subsubsection{Linear Payoffs}

Suppose the payoff function of player $i$ is given by:

$$
\pi_{i}(\mathbf{g})=\sum_{j \in N_{i}(\mathbf{g})} V-\sum_{j \in N} g_{i, j} c_{i} .
$$

Proposition 3: Suppose the payoff function is given by (3) and $\sum_{i \in N} V \geq c$ where $c=\min _{i \in N} c_{i}$. If $\mathbf{g}$ is an efficient network, then it is minimally connected and $2 \leq D(\mathbf{g}) \leq 2 n_{\text {min }}$. Moreover, the efficient network is either a center-sponsored star or a interlinked center-sponsored star.

Proof:The proof is provided in Appendix A.

Observe that if there is a single minimum cost player, then the efficient network is a center-sponsored star. If there is more than one minimum cost player, then the star architecture is still efficient. Galeotti et al. (2006) conclude that the efficient networks and strict Nash networks are the same when a linear payoff specification is assumed. However, if there is more than one minimum cost player in the network, then the interlinked center-sponsored star, where the links are sponsored by the minimum cost players is also efficient. This points out a minor conflict between stability and efficiency since the set of strict Nash networks is a subset of the set of efficient networks.

\footnotetext{
${ }^{3}$ In Example 1, if the cost term is taken as square root instead of square then the efficient network has diameter equal to 2 .
} 
Figure 1 illustrates the possible efficient networks associated with Proposition $3 .^{4}$

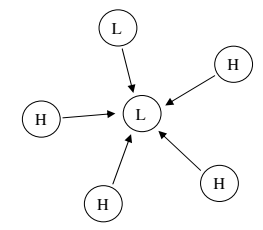

(a) Center-sponsored Star

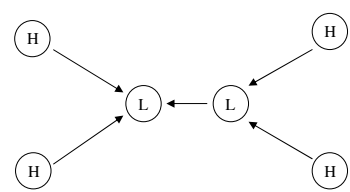

(b) Interlinked Star

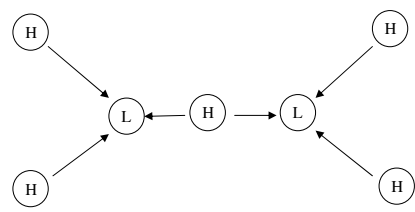

(c) Generalized Interlinked Star

Figure 1: Examples of Efficient Networks

A linear payoff specification is the simplest case and it is possible to identify the diameter and architecture of the efficient network without A1. ${ }^{5}$

\subsubsection{Strictly Convex Payoffs in Cost}

With a linear payoff function, it is possible to determine the diameter and architecture of efficient networks without any strong restrictions. However, the interpretation of the model is limited. Strict convexity specifications are widely used in the literature to address this limitation.

Proposition 4: Suppose the payoff function is given by (1) and $\phi(x, y)$ satisfies strict convexity in cost and A1. If $\mathbf{g}$ is an efficient network, then $\boldsymbol{g}$ is a center-sponsored star, with a minimum cost player as the center of the star.

Proof: The proof is provided in Appendix A.

\subsubsection{Strictly Concave Payoffs in Cost}

It can easily be shown through modifying Example 1 that the diameter can be as high as $(n-1)$ when the payoff function is strictly concave in cost. This result contrasts with the result that is obtained under the strict convexity in costs property. Therefore, an additional condition is imposed to restrict the set of efficient networks.

\footnotetext{
${ }^{4}$ In the below figures, L represents minimum cost players and $\mathrm{H}$ represents high cost players. $\mathrm{H}$ type players can have any cost $c+\epsilon$ where $\epsilon>0$.

${ }^{5}$ Assuming A1 will provide the same result, however note that A1 is stronger than assuming $\sum_{i \in N} V_{i} \geq c$ where $c=\min _{i \in N}\left\{c_{i}\right\}$.
} 
Condition 1: Let $c=\min _{i \in N} c_{i}$. Suppose $\phi(x, y)$ satisfies $\phi(V,(k+1) c)-\phi(V, k c)>\phi\left(V, k^{\prime} c_{i}\right)-$ $\phi\left(V,\left(k^{\prime}-1\right) c_{i}\right) \forall c_{i} \neq c$, and $k \in\{0, \ldots, n-2\}, k^{\prime} \in\{1, \ldots, n-1\}$.

Note that this condition is not on the functional form of the payoffs. Condition 1 eliminates cases where the marginal cost of sponsoring a link for a high cost player is less than the marginal cost of sponsoring a link for a minimum cost player. Once Condition 1 is satisfied, all the links will be sponsored by the minimum cost player(s).

The following lemma provides intuition for the architecture of the efficient networks when the payoff functions are strictly concave in cost.

Lemma 2: Suppose the payoff function is given by (1). Also suppose $\phi(x, y)$ satisfies the strict concavity in the cost argument and satisfies A1. If $n_{\min }>1$, then $\left\|N_{i}(\mathbf{g})|-| N_{j}(\mathbf{g})\right\| \leq 1$ for all $i, j \in S_{\text {min }}$.

Proof: The proof is provided in Appendix.

Proposition 5: Suppose the payoff function is given by (1). Also suppose $\phi(x, y)$ satisfies the strict concavity in the cost argument and satisfies A1 and Condition 1 about the degree of concavity in cost. If $\mathrm{g}$ is an efficient network, then it is minimally connected and $2 \leq D(\mathbf{g}) \leq 2 n_{\text {min }}$. The efficient network is a center-sponsored star for $n_{\min }=1$ and a (generalized) interlinked star for $n_{\min }>1$.

Proof: The proof is provided in Appendix.

Note that by Lemma 2, if there is more than one minimum cost player in $\mathbf{g}$, then a (generalized) interlinked center-sponsored star is the efficient architecture as opposed to the star architecture. The intuition for this case is that it becomes inefficient for a single minimum cost player to sponsor all the links since the payoff is strictly concave in cost. Note that maximal diameter occurs with generalized interlinked star, hence maximal diameter in an efficient network is $2 n_{\min }$. Figure 1(b) and 1(c) provide representatives for this case.

\subsection{Player Heterogeneity in the One-way Flow Model}

In this subsection, the efficient networks in the one-way flow models are identified for player heterogeneity. In the one-way flow model, only the player who sponsors the link accesses the benefit of the link. The model in Galeotti (2006), which is extension of Bala and Goyal (2000) is considered. This 
paper establishes strict Nash networks; however, it gives little information about the efficient networks. The next proposition identifies the efficient network for the player heterogeneous one-way flow model.

Let $\pi_{i}: \mathbf{g} \mapsto \pi_{i}(\mathbf{g})$ be such that $\phi(x, y)$ is strictly increasing in $x$ and strictly decreasing in $y$. Let the payoff function for player $i$ be:

$$
\pi_{i}(\mathbf{g})=\phi\left(V, \sum_{j \in N} g_{i, j} c_{i}\right)
$$

Proposition 6: Suppose the payoff function is given by (4) and Condition (A1) is satisfied. Then the unique efficient architecture is a wheel.

Proof: First, we can use the same arguments as in Proposition 1 to show that an efficient network is minimal. Second, when (A1) is satisfied, we can use the same arguments as in Lemma 1 to show that any efficient network is connected. Therefore, any efficient network is minimally connected. In the one-way flow model, the wheel is the only minimally connected network. The result follows.

In a wheel architecture, sponsoring a single link allows all the agents to access each other and maximizes the total benefit for players with the least amount of cost of link formation. Note that the number of minimum cost players has no impact on the efficient architecture.

\section{Partner Heterogeneity Models}

\subsection{Partner Heterogeneity in the Two-way Flow Model}

\subsubsection{General Payoff Function}

The partner heterogeneity model introduced by Billand et al. (2011) considers a framework where the benefits and costs of link formation vary according to who is being accessed. With this type of heterogeneity, the set of strict Nash networks substantially increases and new architectures arise. This section characterizes the efficient network architectures with partner heterogeneous agents in twoway flow information. Partner heterogeneity allows each player to obtain partner specific cost of link formation. Hence, cost of link formation only depends on the attributes and abilities of the player whom players access. For example, suppose players $i$ and $j$ have a link which allows them to access 
information from each other. Without loss of generality suppose that player $i$ is sponsoring the link. Then, the cost of link formation incurred by $i$ is represented by $c_{j}$.

Proposition 7: Suppose the payoff function is given by (2). Then, any efficient network $\mathbf{g}$ is minimal.

Proof: The proof is similar to proof of Proposition 1, and is omitted.

Let $\pi_{i}: \mathbf{g} \mapsto \pi_{i}(\mathbf{g})$ and $\phi: \mathbb{R}^{2} \rightarrow \mathbb{R}$ such that $\phi(x, y)$ is strictly increasing in $x$ and strictly decreasing in $y$. Suppose that the payoff function is given by (2). The following assumption, A2, is imposed to ensure connectivity in the efficient network.

A2: $\phi\left(x+V, y+g_{i j} c_{j}\right) \geq \phi(x, y)$, for all $j \in N$.

Lemma 3: Suppose the payoff function is given by (2) and A2 is satisfied. Then, any efficient network, $\mathrm{g}$ is minimally connected.

Proof: The proof follows from the proof of Lemma 1, and is omitted.

Proposition 8: Suppose the payoff function is given by (2) and satisfies A2. There exists parameter values $V_{j}$ and $c_{j}$ such that the efficient network, $\mathbf{g}$, has maximum diameter, $(n-1)$.

The proof is omitted since this can easily be shown by modifying Example 1, where $c_{j}$ replaces $c_{i}$. Therefore, additional restrictions are enforced on the payoffs, as in the player heterogeneity model. As before, the linear payoffs, strictly concave and convex payoffs in cost cases are considered.

\subsubsection{Linear Payoffs}

Remark 1 Partner Heterogeneity with Linear Payoffs: The diameter of the efficient architecture for this case is essentially the same as with player heterogeneity with linear payoffs. The main difference between the efficient networks in the player heterogeneity model and the partner heterogeneity model is who sponsors the links. In the player heterogeneity case, the minimum cost players sponsor the links. However, in the partner heterogeneity case, the links are sponsored by non-minimum cost players. Therefore, for $n_{\min }=1$, the efficient network has periphery-sponsored star architecture for the partner heterogeneity case. However, for $n_{\min }>1$, the network might be either star or a generalized interlinked star since both architectures are efficient. 


\subsubsection{Strictly Convex Payoffs in Cost}

For the partner heterogeneity model with strictly convex payoff function, it is possible to construct examples with different architectures with diameter up to $n-1$. This happens especially when there is a substantial variation in the costs of forming links between the players. Therefore, an additional condition is needed in order to characterize the diameter of the network.

Condition 2: Let players $h, l$ be such that $h \in S_{\max }$ and $g_{h, l}=0$. Suppose $\phi(x, y)$ satisfies $\phi\left(V, \sum_{j \in N_{h} \cup\{l\}} c_{j}\right)-\phi\left(V, \sum_{j \in N_{h}} c_{j}\right)>\phi\left(V, \sum_{j \in N_{i} \cup\{l\}} c_{j}\right)-\phi\left(V, \sum_{j \in N_{i}} c_{j}\right) \quad \forall i \in N \backslash S_{\max }$ with $g_{i, l}=0$.

Note that this condition is a very strong assumption and it implies that the marginal cost of sponsoring a link for the highest cost player is less than the marginal cost of sponsoring a link for any other player for the partner heterogeneous models. Once Condition 2 is satisfied, all the links will be sponsored by the highest cost player(s).

Proposition 9: Suppose the payoff function is given by (2) and $\phi(x, y)$ satisfies strict convexity in cost, A2 and Condition 2. If $\mathbf{g}$ is an efficient network, then $2 \leq D(\mathbf{g}) \leq 2 n_{\max }$.

Proof: The proof is provided in Appendix B.

Note that the diameter and the architecture can be different from the player heterogeneity case. If the marginal cost of adding links for a high cost player becomes very little after sponsoring links with the other players, the efficient network can have a center-sponsored star architecture. This is also true when $n_{\max }=1$. However, if $n_{\max }>1$ and the marginal cost of adding links for the highest cost player does not decrease enough, it is possible to have network with diameter at most $2 n_{\max }$.

\subsubsection{Strictly Concave Payoffs in Cost}

Lemma 4: Suppose the payoff function is given by (2). Also suppose $\phi(x, y)$ satisfies the strict concavity in the cost argument and satisfies A2. Then players only sponsor a link with a minimum cost player and the number of links sponsored by each player is at most 1 .

Proof: The proof is provided in Appendix B.

Proposition 10: Suppose the payoff function is given by (2). Also suppose $\phi(x, y)$ satisfies the strict 
concavity in the cost argument and satisfies A2. If $\mathbf{g}$ is an efficient network, then it is minimally connected and $2 \leq D(\mathbf{g}) \leq 1+n_{\min }$. The efficient network is either a periphery-sponsored star or an interlinked periphery-sponsored star.

Proof: The proof is provided in Appendix B.

Note that this architecture is different from the player heterogeneity case. In player heterogeneity, a non-minimum cost player can serve as a bridge player as seen in Figure 1. However, in partner heterogeneity, an efficient network does not have such architecture. Observe that to have a bridge, the player in the player heterogeneity model is not sponsoring any links. These links are sponsored by the minimum cost players around the bridge player. However, such a bridge player in the partner heterogeneity model requires sponsoring two links for that player, which is not efficient when the payoff is strictly concave in cost.

\subsection{Partner Heterogeneity in the One-way Flow Model}

In this subsection, the efficient networks in the one-way flow model are identified, and the model in Billand et al. (2011) is considered.

Remark 2 Partner Heterogeneity in one-way flow model: For this case only the condition to have a connected efficient architecture changes. If $\phi\left(\bar{V}, c_{j}\right)>\phi(V, 0)$ for all $j=1, \ldots, n$ then the unique efficient architecture is a wheel. If $\phi\left(\bar{V}, c_{j}\right)<\phi(V, 0)$ for all $j=1, \ldots, n$ then the empty network is efficient.

Note that the partner heterogeneity setting yields the same result as the player heterogeneity model in the one-way flow model. Again, by sponsoring a single link and incurring the cost $c_{j}$, each player can access all players in the network.

\section{Discussions and Conclusion}

This paper identifies the efficient networks in the player and partner heterogeneity models with various functional forms. There are some notable differences between the efficient architectures under the player and partner heterogeneity models. The first difference is that in the player heterogeneity model, minimum cost player(s) sponsor all the links. However, in partner heterogeneity, a high cost player can also sponsor some links depending on the properties of the payoff function. In the homogeneous 
model discussed by Bala and Goyal (2000), efficient networks have a star architecture. However, when heterogeneity in costs is considered, a rather decentralized efficient architecture in the form of (generalized) interlinked stars can occur. This also yields a change in the diameter of the network. Specifically, when the payoff function is linear in cost, the diameter of the network is always 2 if there is only one minimum cost player, whereas the diameter is at most $2 n_{\min }$ if there is more than one minimum cost player for both player and partner heterogeneous cases. When the payoff function is strictly convex in cost, the diameter of the efficient network is 2 in the player heterogeneous case, whereas for the partner heterogeneous case, $2 \leq D(\mathbf{g}) \leq 2 n_{\max }$. And lastly, for the networks where the payoff function

is strictly concave in cost, the diameter is $2 \leq D(\mathrm{~g}) \leq 2 n_{\min }$ for the player heterogeneous case and $2 \leq D(\mathbf{g}) \leq n_{\min }+1$ for the partner heterogeneous case.

In the one-way flow model, the architecture of the efficient network with player and partner heterogeneity is identical. The only connected efficient architecture is a wheel for these cases, where each agent can access all other agents by sponsoring a single link.

One common property between Nash and efficient networks is that they are both minimal. Galeotti et al. (2006) conclude that Nash and efficient networks coincide if the payoff function is linear in benefits and costs. This is especially true if the number of minimum cost players is one, since the efficient network architecture is a center sponsored star in this case. However, when there is more than one minimum cost player, the set of architectures becomes larger since efficient networks in this case also includes interlinked center-sponsored stars. Billand et al (2011) find that introducing partner heterogeneity increases the set of strict Nash networks and allows to exhibit new architectures such as minimally point contrabasis networks and branching. This holds true for efficiency as well.

Note that not all of the payoff function specifications are comparable in terms of Nash and efficient networks since additional conditions are imposed to identify link formation in some cases.

\section{References}

[1] Bala, V., Goyal, S., (2000a). "A non-cooperative model of network formation", Econometrica, 68, pp. 1181-1229.

[2] Bala, V., Goyal, S., (2000b). "A strategic analysis of network reliability", Rev. Econ. Design, 5, pp. 205-228. 
[3] Billand, P., Bravard, C. and Sarangi, S., (2011). "Strict Nash networks and partner heterogeneity", Int. J. Game Theory, 40, pp. 515-525.

[4] Billand, P., Bravard, C., Sarangi, S. (2012) "Existence of Nash Networks and Partner Heterogeneity". Mathematical Social Sciences 64(2):152-158

[5] Galeotti, A., (2004). "One way flow networks: The role of heterogeneity", Tinbergen Institute Discussion Paper No. 2004-031/1.

[6] Galeotti, A., (2006). "One way flow networks: The role of heterogeneity", Economic Theory, 29, pp. $163-179$

[7] Galeotti, A., Goyal S, Kamphorst J., (2006). "Network formation with heterogeneous players", Games Economic Behavior, 54, pp. 353-372.

[8] Jackson, M., Wolinsky, A., (1996). "A strategic model of social and economic networks", J. Econ. Theory, 71, pp. 44-74.

\section{Appendix A: Player Heterogeneity Model}

Proof of Proposition 3: Suppose the payoff function is given by (3). It is clear that if $\mathbf{g}$ is an efficient network and $\sum_{i \in N} V_{i} \geq c$ then $\mathbf{g}$ is minimally connected. As $n \geq 3$, it follows that $D(\mathbf{g}) \geq 2$. It remains to show that $D(\mathbf{g}) \leq 2 n_{\min }$, and the efficient network is either a center-sponsored star or a interlinked center-sponsored star.

Let $\bar{V}=\sum_{i \in N} V_{i}=n V$ be the total value obtained by each player in the complete network. Since an efficient network is connected, each player obtains a value equal to $\bar{V}$ in an efficient network.

Note that in the centered-sponsored star $\mathbf{g}^{c s s}$, the total costs of links are equal to $(n-1) c$. Toward a contradiction, suppose a minimally connected efficient network $\mathbf{g}$ whose diameter is larger than $2 n_{\min }$. Then there exists in $\mathbf{g}$ a chain $\mathcal{C}_{i, k}$ between two players $i$ and $k$ whose length is strictly larger than $2 n_{\min }$. This chain contains at least a player who is not a minimum cost player. It follows that the total cost of links in $\mathbf{g}$ is strictly larger than $(n-1) c$, and $\mathbf{g}$ is not a minimally connected efficient network, a contradiction.

Now, suppose there are $n_{\min }$ minimum cost players in the network $\mathbf{g}$. Since the payoff function is linear in cost, then the total payoff of the network has the same value if all the links are sponsored by only one of the minimum cost player or each of the minimum cost players sponsor the links. Thus, the 
architecture of the network is either a center-sponsored star or a interlinked center-sponsored star with the minimum cost player(s) at the center.

Proof of Proposition 4: Suppose the payoff function is given by (1) and $(x, y)$ satisfies strict convexity in costs and A1. Suppose that the network $\mathrm{g}$ is efficient.

By Lemma 1, it is clear that $\mathbf{g}$ is minimally connected. Next step is to show that $\mathbf{g}$ is a centersponsored star, with a minimum cost player as the center of the star.

First, since an efficient network is connected, each player obtains a value equal to $\bar{V}=n V$ in an efficient network. Second, wlog let $c_{1}=\min _{j \in N} c_{j}$.

Denote by $\mathbf{g}^{\text {css }}$ the center-sponsored star, with player 1 as the center of the star. Suppose a network $\mathbf{g}^{0} \neq \mathbf{g}^{\text {css }}$. Then, we show that $\mathbf{g}^{\text {css }}$ yields more aggregate payoff than $\mathbf{g}^{0}$.

Let the vector $\left(\left|N_{1}^{0}\right|,\left|N_{2}^{0}\right|, \ldots,\left|N_{n}^{0}\right|\right)$ be the vector of the number of links formed by the players in $\mathbf{g}^{0}$, with $\left|N_{i}^{0}\right|$ the number of links formed by player $i$ in $\mathbf{g}^{0}$.

The following process is built. In this process, $\mathbf{N}^{t}=\left(\left|N_{1}^{t}\right|,\left|N_{2}^{t}\right|, \ldots,\left|N_{n}^{t}\right|\right)$ is the vector of number of links obtained at the end of Step $t$, with $\left|N_{i}^{t}\right|$ the number of links formed by player $i$ at the end of Step $t$.

\section{Step 1:}

Since $\mathbf{g}^{0} \neq \mathbf{g}^{c s s}$, then $\left|N_{i}^{0}\right|>0$ for at least one player $i \neq 1$. Let $i_{1} \neq 1$ be a player such that $\left|N_{i_{1}}\left(\mathbf{g}^{0}\right)\right|>0$.

Let $\mathbf{N}^{1}$ be such that $\left|N_{1}^{1}\right|=\left|N_{1}^{0}\right|+\left|N_{i_{1}}^{0}\right|$ and $\left|N_{i_{1}}^{1}\right|=0$ and $\left|N_{i}^{1}\right|=\left|N_{i}^{0}\right|$ for all $i \neq 1, i_{1}$.

$$
\begin{aligned}
\phi\left(\bar{V},\left|N_{1}^{1}\right| c_{1}\right)+\phi\left(\bar{V},\left|N_{i_{1}}^{1}\right| c_{i_{1}}\right) & =\phi\left(\bar{V},\left(\left|N_{1}^{0}\right|+\left|N_{i_{1}}^{0}\right|\right) c_{1}\right)+\phi(\bar{V}, 0) \\
& >\Phi\left(\bar{V},\left|N_{1}^{0}\right| c_{1}\right)+\phi\left(\bar{V},\left|N_{i_{1}}^{0}\right| c_{1}\right) \\
& \geq \phi\left(\bar{V},\left|N_{1}^{0}\right| c_{1}\right)+\phi\left(\bar{V},\left|N_{i_{1}}^{0}\right| c_{i_{1}}\right)
\end{aligned}
$$

The first inequality comes from the convexity of $\phi$ with regard to its second argument, while the second inequality is due to the decreasing of $\phi$ in its second argument.

If $\mathbf{g}^{\text {css }}$ is the network associated with $\mathbf{N}^{1}$, then the process stops. Otherwise we go to Step 2.

\section{Step 2:}

Let $i_{2} \neq 1, i_{1}$ be a player such that $\left|N_{i_{2}}^{1}\right|>0$. Let $\mathbf{N}^{2}$ be such that $\left|N_{1}^{2}\right|=\left|N_{1}^{1}\right|+\left|N_{i_{2}}^{1}\right|$ and $\left|N_{i_{2}}^{2}\right|=0$ 
and $\left|N_{i}^{2}\right|=\left|N_{i}^{1}\right|$ for all $i \neq 1, i_{2}$. By the same argument as in Step 1, it can be shown that

$$
\begin{aligned}
\phi\left(\bar{V},\left|N_{1}^{2}\right| c_{1}\right)+\phi\left(\bar{V},\left|N_{i_{2}}^{2}\right| c_{i_{2}}\right) & =\phi\left(\bar{V},\left(\left|N_{1}^{1}\right|+\left|N_{i_{2}}^{1}\right|\right) c_{1}\right)+\phi(\bar{V}, 0) \\
& >\Phi\left(\bar{V},\left|N_{1}^{1}\right| c_{1}\right)+\phi\left(\bar{V},\left|N_{i_{2}}^{1}\right| c_{i_{2}}\right) .
\end{aligned}
$$

If $\mathbf{g}^{\text {css }}$ is the network associated with $\mathbf{N}^{2}$, then the process stops. Otherwise we go to Step 3. Since $n$ is finite there exists a number $k$ such that $\mathbf{g}^{c s s}$ is the network associated with $\mathbf{N}^{k}$.

Now, it remains to show that $W\left(\mathbf{g}^{c s s}\right)-W\left(\mathbf{g}^{0}\right)=W\left(\mathbf{g}^{k}\right)-W\left(\mathbf{g}^{0}\right)>0$.

First note that

$$
\phi\left(\bar{V}, N_{1}^{t} c_{1}\right)-\phi\left(\bar{V}, N_{1}^{t-1} c_{1}\right)+\phi(\bar{V}, 0)-\phi\left(\bar{V},\left|N_{i_{t}}^{t-1}\right| c_{k}\right)>0
$$

for all $1 \leq t \leq k$.

Moreover,for player $1, \phi\left(\bar{V},\left|N_{1}^{k}\right| c_{1}\right)=\phi\left(\bar{V},(n-1) c_{1}\right)$, and for each player $i \neq 1$, they payoffs are $\phi\left(\bar{V},\left|N_{i}^{k}\right| c_{i}\right)=\phi(\bar{V}, 0)$. Therefore, it follows that:

$$
\begin{aligned}
W\left(\mathbf{g}^{c s s}\right)-W\left(\mathbf{g}^{0}\right)= & \phi\left(\bar{V},(n-1) c_{1}\right)-\phi\left(\bar{V},\left|N_{1}^{0}\right| c_{1}\right)+\sum_{i_{t}:\left|\mathbf{g}^{\text {gss }}\right| \neq\left|N_{i}^{0}\right|}\left(\phi(\bar{V}, 0)-\phi\left(\bar{V},\left|N_{i_{t}}^{0}\right| c_{t}\right)\right) \\
= & {\left[\phi\left(\bar{V},(n-1) c_{1}\right)-\phi\left(\bar{V},\left|N_{1}^{k-1}\right| c_{1}\right)\right]+\left[\phi\left(\bar{V},\left|N_{1}^{k-1}\right| c_{1}\right)-\phi\left(\bar{V},\left|N_{1}^{k-2}\right| c_{1}\right)\right]+\ldots+} \\
& {\left[\phi\left(\bar{V},\left|N_{1}^{1}\right| c_{1}\right)-\phi\left(\bar{V},\left|N_{1}^{0}\right| c_{1}\right)\right]+\sum_{i_{t}:\left|N^{c s s}\right| \neq\left|N_{i}^{0}\right|}\left(\phi(\bar{V}, 0)-\phi\left(\bar{V},\left|N_{i_{t}}^{t-1}\right| c_{t}\right)\right) } \\
= & {\left[\phi\left(\bar{V},(n-1) c_{1}\right)-\phi\left(\bar{V},\left|N_{1}^{k-1}\right| c_{1}\right)+\phi(\bar{V}, 0)-\phi\left(\bar{V},\left|N_{i_{k}}^{k-1}\right| c_{k}\right)\right]+\ldots+} \\
& {\left[\phi\left(\bar{V},\left|N_{1}^{1}\right| c_{1}\right)-\phi\left(\bar{V},\left|N_{1}^{0}\right| c_{1}\right)+\phi(\bar{V}, 0)-\phi\left(\bar{V},\left|N_{i_{k}}^{0}\right| c_{i_{1}}\right)\right] } \\
> & 0
\end{aligned}
$$

since each term of the last sum is positive.

This concludes that the efficient network is the center-sponsored star, with player 1 as the center of the star.

Proof of Lemma 2: Suppose $n_{\min }>1$ and let $\mathbf{g}^{0}$ be an efficient network. First, since an efficient network is connected, each player obtains a value $\bar{V}=n V$ in $\mathbf{g}^{0}$. Second, let players 1 and 2 belong to 
$S_{\text {min }}$, with $c_{1}=c_{2}=c$, and suppose wlog that $\left|N_{1}^{0}\right|=\left|N_{2}^{0}\right|+2$. Suppose a network $\mathbf{g}^{1}$ where one of the links sponsored by player $1 \mathrm{in} \mathbf{g}^{0}$ is now sponsored by player 2. i.e., $\left|N_{1}^{1}\right|=\left|N_{1}^{0}\right|-1, \quad\left|N_{2}^{1}\right|=\left|N_{2}^{0}\right|+1$, and $\left|N_{i}^{1}\right|=\left|N_{i}^{0}\right|$ for all $i \neq 1,2$. We have

$$
\begin{aligned}
W\left(\mathbf{g}^{1}\right)-W\left(\mathbf{g}^{0}\right) & =\phi\left(\bar{V},\left|N_{1}^{1}\right| c_{1}\right)+\phi\left(\bar{V},\left|N_{2}^{1}\right| c_{2}\right)-\phi\left(\bar{V},\left|N_{1}^{0}\right| c_{1}\right)-\phi\left(\bar{V},\left|N_{2}^{0}\right| c_{2}\right) \\
& =\phi\left(\bar{V},\left(\left|N_{1}^{0}\right|-1\right) c_{1}\right)+\phi\left(\bar{V},\left(\left|N_{2}^{0}\right|+1\right) c_{2}\right)-\phi\left(\bar{V},\left|N_{1}^{0}\right| c_{1}\right)-\phi\left(\bar{V},\left|N_{2}^{0}\right| c_{2}\right) \\
& =\phi\left(\bar{V},\left(\left|N_{2}^{0}\right|+1\right) c_{1}\right)+\phi\left(\bar{V},\left(\left|N_{2}^{0}\right|+1\right) c_{2}\right)-\phi\left(\bar{V},\left(\left|N_{2}^{0}\right|+2\right) c_{1}\right)-\phi\left(\bar{V},\left|N_{2}^{0}\right| c_{2}\right) \\
& =\left[\phi\left(\bar{V},\left(\left|N_{2}^{0}\right|+1\right) c\right)-\phi\left(\bar{V},\left|N_{2}^{0}\right| c\right)\right]-\left[\phi\left(\bar{V},\left(\left|N_{2}^{0}\right|+2\right) c\right)-\phi\left(\bar{V},\left(\left|N_{2}^{0}\right|+1\right) c\right)\right] \\
& >0
\end{aligned}
$$

where last inequality is because $\phi$ is decreasing and strictly concave in the cost argument.

Note that using the same method as above, one can show that $W\left(\mathbf{g}^{k-1}\right)>W\left(\mathbf{g}^{0}\right)$ for $\left|N_{1}^{0}\right|=\left|N_{2}^{0}\right|+k$ where $k \geq 2$.

Proof of Proposition 5: Suppose the payoff function is given by $(2)$ and $\phi(x, y)$ satisfies strict concavity in costs, A1 and Condition 1, and let $\mathbf{g}^{0}$ be an efficient network. By Lemma $1, \mathbf{g}^{0}$ is minimally connected. Since $n \geq 3, D\left(\mathbf{g}^{0}\right) \geq 2$.

To obtain a contradiction, suppose $D\left(\mathbf{g}^{0}\right) \geq 2 n_{\min }+1$, and let $c_{1}=\min _{j \in N} c_{j}$. Since $D\left(\mathbf{g}^{0}\right) \geq 2 n_{\min }+1$, there is a player $i_{1} \notin S_{\text {min }}$ such that $\left|N_{i_{1}}\left(\mathbf{g}^{0}\right)\right|>0$, and a player $j \neq i_{1}$ such that $i_{1} j \in \mathbf{g}^{0}$. Either (i) $j \in S_{\min }$ or (ii) $j \notin S_{\min }$.

(i) Suppose $j \in S_{\min }$. Then if we replace the link $i_{1} j$ by the link $j i_{1}$, by Condition 1 the sum of the payoffs of player $i_{1}$ and $j$ increases while the payoffs of the other players do not change. Hence $\mathbf{g}^{0}$ is not efficient, a contradiction.

(ii) Suppose $j \notin S_{\text {min }}$. Since $\mathbf{g}^{0}$ is minimally connected, there exists a player $k \in S_{\text {min }}$ such that $k \notin N_{i_{1}}\left(\mathbf{g}^{0}-i_{1} j\right)$ or $k \notin N_{j}\left(\mathbf{g}^{0}-i_{1} j\right)$. In the first case, if we replace the link $i_{1} j$ by the $k i_{1}$, the network will still be connected and the players will obtain the same value. Moreover, by Condition 1 the sum of the payoffs of player $i_{1}$ and $k$ increases while the payoffs of the other players do not change. Hence $\mathbf{g}^{0}$ is not efficient, a contradiction. In the second case, if we replace the link $i_{1} j$ by the link $k j$, then we obtain the same result.

Now, suppose there are $n_{\min }$ minimum cost players in the network $\mathbf{g}$. If $n_{\min }=1$, then $\mathbf{g}$ is center- 
sponsored star with the minimum cost player as the center of the star. However, if $n_{\min }>1$, then by Lemma 2, the architecture of $\mathbf{g}$ becomes a generalized interlinked center-sponsored star where a high cost player can stay in the center as a bridge player without sponsoring any links; see Figure 1(c).

\section{Appendix B: Partner Heterogeneity Model}

Proof of Lemma 4: Let $\mathbf{g}^{0}$ be an efficient network. In what follows, let $\mathbf{g}^{1}=\mathbf{g}^{0}-\sum_{\ell \in N_{i}} i \ell+i j$. Since $\mathrm{g}^{0}$ is minimally connected, it is a tree.

(i) We begin to prove that players only sponsor links with minimum cost players. To introduce a contradiction suppose there exists a player $i$ in $\mathbf{g}^{0}$ who has formed a link with a player $j \notin S_{\min }$.

First suppose $j$ is a leaf of the tree $\mathbf{g}^{0}$. We have $c_{i} \geq c_{j}$, otherwise by concavity, we have $W\left(\mathbf{g}^{0}-i, j+\right.$ $j, i)>W\left(\mathbf{g}^{0}\right)$, and $\mathbf{g}^{0}$ is not an efficient network, a contradiction. Therefore $i \notin S_{\text {min }}$. Then there exists a player $k \neq i, j$ such that $k \in S_{\min }$. By concavity we have $W\left(\mathbf{g}^{0}-i, j+j, k\right)>W\left(\mathbf{g}^{0}\right)$, and $\mathbf{g}^{0}$ is not an efficient network, a contradiction.

Second, suppose $j$ is a not leaf of the tree $\mathbf{g}^{0}$. There are two cases:

Case $1 . i$ is a leaf of the tree $\mathbf{g}^{0}$. We have $i \notin S_{\text {min }}$, otherwise by concavity we have $W\left(\mathbf{g}^{0}-i, j+j, i\right)>$ $W\left(\mathbf{g}^{0}\right)$, and $\mathbf{g}^{0}$ is not an efficient network, a contradiction. Therefore there exists a player $k \neq i, j$ such that $k \in S_{\text {min }}$. However, we have $W\left(\mathbf{g}^{0}-i, j+i, k\right)>W\left(\mathbf{g}^{0}\right)$, and $\mathbf{g}^{0}$ is not an efficient network, a contradiction.

Case 2. $i$ and $j$ are not leaves of the tree $\mathbf{g}^{0}$.

Note that for all players $k \in N_{i}\left(\mathbf{g}^{1}\right)$, we have $k \notin S_{\text {min }}$. Otherwise, we have $W\left(\mathbf{g}^{0}-i, j+i, k\right)>W\left(\mathbf{g}^{0}\right)$, and $\mathbf{g}^{0}$ is not an efficient network, a contradiction.

Let $\ell \in S_{\text {min }}$. We know that we have $\ell \notin N_{1}\left(\mathbf{g}^{1}\right)$. Let $k \in N_{1}\left(\mathbf{g}^{1}\right)$ be a leaf of the tree $\mathbf{g}^{0}$. There exists a player $k^{\prime} \in N_{1}\left(\mathbf{g}^{1}\right)$ such that $g_{k, k^{\prime}}=1$ or $g_{k^{\prime}, k}=1$.

Suppose $g_{k, k^{\prime}}=1$. Since $c_{k^{\prime}}>c_{\ell}$, we have $W\left(\mathbf{g}^{0}-k, k^{\prime}+k, \ell\right)>W\left(\mathbf{g}^{0}\right)$, and $\mathbf{g}^{0}$ is not an efficient network, a contradiction.

Suppose $g_{k^{\prime}, k}=1$. Since $c_{k^{\prime}}>c_{\ell}$, and $N_{k^{\prime}}\left(\mathbf{g}^{0}\right) \geq N_{k}\left(\mathbf{g}^{0}\right)$, by concavity, we have $W\left(\mathbf{g}^{0}-k^{\prime}, k+k, \ell\right)>$ $W\left(\mathbf{g}^{0}\right)$, and $\mathbf{g}^{0}$ is not an efficient network, a contradiction.

The result follows. 
(ii) We now show that $\left|N_{i}\left(\mathbf{g}^{0}\right)\right| \leq 1$ for all $i$ in $\mathrm{N}$. To introduce a contradiction, wlog, suppose there exists in $\mathbf{g}^{0}$ a player $i$ that has formed two links, and let players $j, j^{\prime}$ such that $g_{i, j}=1$, and $g_{i, j^{\prime}}=1$. To show that $\mathbf{g}^{0}$ is not efficient, we consider a player $k$ who is a leaf of the tree $\mathbf{g}^{0}$ such that $k \in N_{i}\left(\mathbf{g}^{1}\right)$ and a player $t$ such that $g_{t, k}=1$ or $g_{k, t}=1$.

Suppose $g_{t, k}=1$. Since, $\left|N_{k}\left(\mathbf{g}^{0}\right)\right|<\left|N_{i}\left(\mathbf{g}^{0}\right)\right|$, by concavity we have $W\left(\mathbf{g}^{0}-i, j+k, j\right)>W\left(\mathbf{g}^{0}\right)$, and $\mathbf{g}^{0}$ is not an efficient network, a contradiction.

Suppose $g_{k, t}=1$. Since $g_{i, j}=1$, there is a player $\ell$ who belongs to a chain between $k$ and $i$ who has not formed any link in $\mathbf{g}^{0}$. Since $j^{\prime} \in S_{\text {min }}$, by concavity we have $W\left(\mathbf{g}^{0}-i, j+\ell, j^{\prime}\right)>W\left(\mathbf{g}^{0}\right)$, and $\mathbf{g}^{0}$ is not an efficient network, a contradiction.

Proof of Proposition 9: Note that this proof follows similar steps as in the proof of the Proposition 5. Suppose the payoff function is given by $(2)$ and $\phi(x, y)$ satisfies strict convexity in cost, A2 and Condition 2, and let $\mathbf{g}^{0}$ be an efficient network. By Lemma 3, it is clear that $\mathbf{g}^{0}$ is minimally connected. Since $n \geq 3$, it follows that $D\left(\mathbf{g}^{0}\right) \geq 2$. Now, we want to prove that $D(\mathbf{g}) \leq 2 n_{\max }$.

Suppose $D\left(\mathbf{g}^{0}\right) \geq 2 n_{\max }+1$. Then there is at least one player $i_{1} \notin S_{\max }$ such that $\left|N_{i_{1}}\left(\mathbf{g}^{0}\right)\right|>0$, and a player $j \neq i_{1}$ such that $i_{1} j \in \mathbf{g}^{0}$. Either (i) $j \in S_{\max }$ or (ii) $j \notin S_{\max }$.

(i) Suppose $j \in S_{\max }$. Then if we replace the link $i_{1} j$ by the link $j i_{1}$, by Condition 2 the sum of the payoffs of players $i_{1}$ and $j$ increases while the payoffs of the other players do not change. Hence $\mathbf{g}^{0}$ is not efficient, a contradiction.

(ii) Suppose $j \notin S_{\max }$. Since $\mathbf{g}^{0}$ is minimally connected. Thus, the network $\mathbf{g}^{0}-i_{1} j$ is a disconnected network with two components. There exists a player $k \in S_{\max }$ such that $k \notin N_{i_{1}}\left(\mathbf{g}^{0}-i_{1} j\right)$ or $k \notin$ $N_{j}\left(\mathrm{~g}^{0}-i_{1} j\right)$. In the first case, if we replace the link $i_{1} j$ by the $k i_{1}$, the network will still be connected and the players will obtain the same value. Moreover, by Condition 2 the sum of the payoffs of player $i_{1}$ and $k$ increases while the payoffs of the other players do not change. Hence $\mathbf{g}^{0}$ is not efficient, a contradiction. In the second case, if we replace the link $i_{1} j$ by the link $k j$, then we obtain the same result.

Thus, the above cases show that the $\mathbf{g}^{0}$ where $D\left(\mathbf{g}^{0}\right) \geq 2 n_{\max }+1$ is not an efficient network.

In the case of $n_{\max }=1$, the

Proof of Proposition 10: Suppose the payoff function is given by (2). Also suppose $\phi(x, y)$ satisfies 
the strict concavity in the cost argument and satisfies A2. By Lemma 3, it is clear that $\mathbf{g}$ is minimally connected. Since $n \geq 3$, it follows that $D(\mathbf{g}) \geq 2$. If $n_{\min }=1$, then by Lemma 4 , the unique efficient architecture is periphery-sponsored star. Now, it remains to show that $D(\mathbf{g}) \leq n_{\min }+1$ and the architecture is either periphery-sponsored star or interlinked periphery-sponsored star.

Suppose, on the contrary that $D(\mathrm{~g}) \geq n_{\min }+2$, then there exists a player such that either she acts as a bridge and sponsored two links or she sponsors a link with a player outside of $S_{\min }$. The former case is not possible since, by Lemma 4, each player sponsors at most one link. Moreover, the latter case is not possible since again by Lemma 4 , the players only sponsors links with players who have minimum cost of forming links. Therefore, a contradiction is obtained with an efficient network with diameter greater than $n_{\min }+1$. 